\title{
A01-28106
}

\section{AIAA 2001-1761}

Velocity Measurement in a Dual-mode Supersonic Combustor using Particle Image Velocimetry

C.P. Goyne, J.C. McDaniel, R.H. Krauss and S.W. Day Aerospace Research Laboratory

University of Virginia

Charlottesville, Virginia 22904 USA

\footnotetext{
AIAA/NAL-NASDA-ISAS $10^{\text {th }}$ International Space Planes and Hypersonic Systems and Technologies Conference 24-27 April 2001 / Kyoto, Japan
} 


\title{
VELOCITY MEASUREMENT IN A DUAL-MODE SUPERSONIC COMBUSTOR USING PARTICLE IMAGE VELOCIMETRY
}

\author{
C.P. Goyne", J.C. McDaniel ${ }^{\dagger}$, R.H. Krauss ${ }^{\ddagger}$ and S.W. Day ${ }^{\$}$ \\ University of Virginia, Charlottesville, Virginia USA
}

\begin{abstract}
Temporally and spatially-resolved, two-component measurements of velocity in a supersonic hydrogen-air combustor are reported. The combustor had a single unswept ramp fuel injector and operated with an inlet Mach number of 2 and a flow total temperature approaching $1200 \mathrm{~K}$. The experiment simulated the mixing and combustion processes of a dual-mode scramjet operating at a flight Mach number near 5 . The velocity measurements were obtained by seeding the fuel with alumina particles and performing Particle Image Velocimetry on the mixing and combustion wake of the ramp injector. To assess the effects of combustion on the fuel air-mixing process, the distribution of time-averaged velocity and relative turbulence intensity was determined for the cases of fuel-air mixing and fuel-air reacting. Relative to the mixing case, the near field core velocity of the reacting fuel jet had a slower streamwise decay. In the far field, downstream of 4 to 6 ramp heights from the ramp base, the heat release of combustion resulted in decreased flow velocity and increased turbulence levels. The reacting measurements were also compared with a computational fluid dynamics solution of the flow field. Numerically predicted velocity magnitudes were higher than that measured and the jet penetration was lower.
\end{abstract}

\section{Introduction}

Modern computational fluid dynamics (CFD) is becoming an increasingly important tool for the design of dual-mode scramjets. Because of the complexity of the combustor flow-field, however, CFD is still regarded as unreliable in terms of flight performance predictive capabilities. For example, for NASA's Hyper-X vehicle, which incorporates an air-frame integrated dual-mode scramjet, experimental data and analytical analysis, rather than CFD, have been identified as the primary tools for engine performance predictions and flight test risk reduction ${ }^{1}$. One way to improve CFD capabilities is to validate the numerical models against increasingly comprehensive bench-mark data sets. As a means of obtaining such a data set, an experimental study of a model dual-mode scramjet combustor has been undertaken at the University of Virginia-4. Using conventional instrumentation and multiple laser-based diagnostic techniques, the study aims to further the understanding of the fuel-air mixing and combustion processes within the combustor and to resolve the uncertainty of CFD predictions of such

\footnotetext{
- Research Associate, Member AIAA

† Professor, Senior Member AIAA

${ }^{\ddagger}$ Research Consultant

Graduate Research Assistant, Student member AIAA.

Aerospace Research Laboratory, University of Virginia, 570 Edgemont Rd, Charlottesville, VA, 22904 USA

Copyright $\odot 2001$ by the authors. Published by the

American Institute of Aeronautics and Astronautics, Inc. with permission.
}

flows. Of particular interest to the present study is the measurement of flow velocity within the combustor. Velocity data is an important parameter for combustor analysis and CFD validation; however, the quantity rarely is featured in dual-mode data sets because of the difficulties of accurately measuring velocity in the high speed and high temperature flow of supersonic combustors.

The flow field of a dual-mode combustor is highly three dimensional ${ }^{2,5}$ and therefore, the planar measurement of velocity in two or even three components is highly suited to dual-mode CFD validation. There are two broad types of optical methods that are particularly suited to such planar measurements. The first is measuring the Doppler shift of light produced by laserinduced fluorescence (LIF) ${ }^{4}$ or the Doppler shift of Rayleigh ${ }^{6}$ or $\mathrm{Mie}^{7.8}$ scattered light from natural or seeded molecules or particles. The second method is to measure displacement of illuminated particles in the flow using a method known as Particle Image Velocimetry (PIV). Of these techniques, only a member of the first group has been successfully applied to scramjet combustion. Gauba et al. ${ }^{4}$ used the Doppler shift of $\mathrm{OH}$ laser induced fluorescence (LIF) to measure two components of velocity in a model dual-mode combustor. Good results were obtained; however, the technique was only capable of generating time-averaged measurements and, hence, could not be used to look at instabilities in the flow and quantities such as turbulence intensity. Also, the presence of $\mathrm{OH}$ (generated by the combustion process) was required and, therefore, measurements could not be performed on

1

American Institute of Aeronautics and Astronautics 
the fuel jet itself or where $\mathrm{OH}$ concentrations were low. Like all Doppler shifted methods, the use of a single camera necessitated the use of one laser sheet per velocity component (or similarly for a single laser sheet, one camera per velocity component) and hence, the technique was hardware intensive and required a test section with good optical access (at least three windows for the measurement of two components) ${ }^{10}$.

Particie image velocimetry, however, is particularly suited to the interrogation of fuel-air mixing and combustion in a dual-mode combustor. The technique involves seeding a region of flow with particles and determining the particle displacement, and hence velocity, by imaging the particles while illuminated via two temporally separated laser pulses. The technique is capable of generating both instantaneous and timeaveraged, spatially-resolved velocity measurements in two and even three components. Practically, PIV is a well established technique and systems are commercially available and are readily implemented. The technique requires less optical access than methods that employ a Doppler shift. For two or three component measurements, PIV requires one or two less windows, respectively, than for the Doppler shifted technique of Particle Doppler Velocimetry (PDV) ${ }^{10}$, for example. Although PDV has the potential of providing better spatial resolution and dynamic range than PIV, PIV requires a lower density of seed particles ${ }^{10}$. This makes the technique better suited to high speed combustion applications where seeding density is limited due to seeder mass flux limitations and is reduced due to the flow density reduction of combustion.

PIV has existed in its modern digital form (employing electronic image capture and computer based postprocessing) since the early $1990^{\prime} \mathrm{s}^{11}$. The technique has been extensively applied to low speed fluid flows ${ }^{9}$ and recently to the study of high speed flows ${ }^{10}$. PIV has been applied to supersonic ${ }^{12,13}$ and hypersonic flows ${ }^{14}$ and speeds as high as $1500 \mathrm{~m} / \mathrm{s}$ have been measured ${ }^{13}$. In such high speed flows, however, particle momentum is high and particles do not accurately track the flow in and immediately downstream of regions of high flow acceleration or deceleration. However, as discussed later, the extent of flow tracking downstream of a shock wave, for example, can be predicted. PIV has been applied to subsonic combustion cases ${ }^{15}$, and to simulated fuel-air mixing studies in which the freestream was supersonic ${ }^{16}$. However, the measurement of velocity using PIV in a supersonic combustor or dual-mode combustor has not been reported.
The present contribution reports on PIV measurements in a model dual-mode scramjet combustor. The experiment consisted of the direct connect test of a Mach 2 hydrogen-air burner with a single $10^{\circ}$ unswept ramp fuel injector. Alumina particles were introduced into the hydrogen stream prior to injection into the combustor and PIV was performed on the mixing and combustion wake downstream of the fuel injector. The test flow conditions of the experiment simulated a flight Mach number of 5 . The paper begins by describing the combustor configuration, flow conditions and PIV technique. In particular, the seeding, imaging and data processing aspects and experimental uncertainty of the PIV technique are discussed. Spatially-resolved, twocomponent velocity measurements along the centerplane of the combustor are then presented. In order to investigate the effects of combustion on the fuel-air mixing process, velocity measurements are presented in terms of non-reacting fuel-air (mixing only) and reacting fuel-air. A numerical simulation of the combustion process is also compared with the reacting results.

\section{Experiment}

\section{Combustor configuration}

The configuration of the model dual-mode combustor was the same as previously used for other benchmarking experiments ${ }^{2-4}$. A schematic of the configuration is presented in Fig. 1. The combustor essentially consisted of a Mach 2 nozzle, a short constant area rectangular isolator and a rectangular combustion duct. A single $10^{\circ}$ unswept ramp fuel injector was located on one of the walls of the combustor. This wall is referred to here as the injection wall. Hydrogen fuel was injected into the combustor via a Mach 1.7 conical nozzle that was located within the ramp base. The nozzle centerline was parallel to the $10^{\circ}$ compression surface of the ramp.

Dimensions of the combustor are presented in Table 1. As with other linear dimensions reported here, each has been normalized by the height of the ramp injector, perpendicular to the injection wall $(\mathrm{H}=6.35 \mathrm{~mm})$. The combustion duct was of constant cross sectional area up to $10 \mathrm{H}$ downstream of the base of the ramp. Between this point and $58 \mathrm{H}$, where the combustor exited to atmosphere as a free jet, the injection wall had a divergence of $2.9^{\circ}$.

Thirty static pressure taps extended axially along the injection wall between $-7 \mathrm{H}$ and $56 \mathrm{H}$. The taps were located along the axis of symmetry of the combustor (except for two taps at $4 \mathrm{H}$, which were $\pm 1 \mathrm{H}$ from the centerline). Pressure was measured using a Setra ${ }^{\mathrm{TM}}$

2

American Institute of Aeronautics and Astronautics 
pressure transducer and a scanning Scanivalve ${ }^{*}$. The scanner operated at a tap interrogation frequency of 0.5 Hz. Three thermocouples were also located along the centerline of the injection wall. These were located at $7 \mathrm{H}, 10 \mathrm{H}$ and $22 \mathrm{H}, 0.1 \mathrm{H}$ beneath the surface of the wall. Experimental uncertainty in measured pressure and temperature was estimated to be $\pm 2 \%$.

Optical access to the combustor was facilitated via three fused silica windows. An observation window was located on the wall facing the injection wall and the remaining windows were each located in one of the side walls. The Mach 2 nozzle, window support frames and portions of the combustion duct were water cooled. Between the axial locations of $-8 \mathrm{H}$ and $26 \mathrm{H}$, the injection wall and fuel injection ramp were zirconia coated.

Table 1. Combustor geometry dimensions

\begin{tabular}{lc}
\multicolumn{1}{c}{ Feature } & Dimension \\
\hline Ramp height & $\mathrm{H}=6.35 \mathrm{~mm}$ \\
$\begin{array}{l}\text { Combustion duct inlet and isolator } \\
\text { height }\end{array}$ & $4 \mathrm{H}$ \\
Combustion duct and isolator & $6 \mathrm{H}$ \\
width & \\
Isolator length & $2 \mathrm{H}$ \\
X distance to start of $2.9^{\circ}$ & $10 \mathrm{H}$ \\
divergence & \\
X extent of combustion duct & $58 \mathrm{H}$ \\
Ramp width & $2 \mathrm{H}$ \\
Ramp compression angle & $10^{\circ}$ \\
Injector exit Ylocation & $0.65 \mathrm{H}$ \\
Injector port diameter & $0.4 \mathrm{H}$ \\
Injection angle & $10^{\circ}$ \\
\hline
\end{tabular}

\section{Facility and flow conditions}

The experiments were performed using the Supersonic Combustion Tunnel Facility at the University of Virginia. The facility, which is fully described elsewhere ${ }^{17,18}$, consisted of an oil-free compressor which supplied air, via desiccant dryers, to outdoor storage tanks. The tanks in turn supplied an indoor high pressure vessel which contained a fourteen-stage electrical resistance heater. Heated test gas then passed through a ceramic flow straightener before supplying the dual-mode combustor. The test flow and combustion products exited the building via an open-ended exhaust pipe. The facility was capable of continuous, steadystate operation and could achieve a flow total temperature near $1200 \mathrm{~K}$ with a test flow static pressure of 0.5 atmosphere at Mach 2. Unlike vitiated and arc driven facilities, the electrical resistance heater supplied a test flow free of contaminants such as water, NO, particles and radical species ${ }^{17,18}$.

Air and fuel test flow conditions are presented in Table 2. The combustor was operated at an equivalence ratio that ensured supersonic inflow to the isolator ${ }^{2}$. This equivalence ratio was determined using a measured mass flow rate of hydrogen and a mass flow rate of air that was calculated using measured stagnation properties and an assumed facility discharge coefficient of 0.99. Following facility warm up, tests were generally conducted over a one hour period (limited only by fuel supply). During this period, air and fuel total conditions were typically maintained to within $2 \%$ of nominal values. As the flow static temperature at Mach 2 was below the autoignition temperature of hydrogen, a hydrogen-oxygen detonation-driven igniter system was used for initial ignition of the combustor. Combustion was then self sustaining following ignition.

Table 2. Test conditions

\begin{tabular}{llll}
\hline Parameter & Air & Fuel & Error \\
\hline Total pressure (kPa) & 390 & 1010 & $\pm 3 \%$ \\
Total temperature (K) & 1120 & 300 & $\pm 3 \%$ \\
Mach number" $^{2}$ & 2.1 & 1.7 & \\
Static pressure" (kPa) & 47 & 210 & \\
Static temperature" $(\mathrm{K})$ & 650 & 190 & \\
Velocity" (m/s) & 1030 & 1760 & \\
Equivalence ratio & & 0.28 & $\pm 5 \%$ \\
Property at nozzle exit determined using nozzle area \\
ratio and assuming isentropic flow \\
\multicolumn{4}{l}{} \\
PIV technique
\end{tabular}

Seeding

The seed material adopted for the present study consisted of alumina $\left(\mathrm{Al}_{2} \mathrm{O}_{3}\right)$ powder with a primary particle diameter near $0.3 \mu \mathrm{m}$ and density of 3940 $\mathrm{kg} / \mathrm{m}^{3}$. Micron and sub-micron alumina particles have previously been used for PIV studies of high speed ${ }^{14}$ and combusting flows ${ }^{15}$. The present particles had a melting and boiling point near $2350 \mathrm{~K}$ and $3250 \mathrm{~K}$, respectively. Previous CFD analysis ${ }^{2}$ of the model dualmode combustor indicated that a maximum flow temperature of $2380 \mathrm{~K}$ was expected in the measurement region of interest. Particle breakdown was, therefore, expected to be minimal.

When stored, the alumina particles tend to form agglomerates that have effective diameters in the range of 2 to $40 \mu \mathrm{m}$. In order to ensure adequate flow tracking

3

American Institute of Aeronautics and Astronautics 
the agglomerates need to be broken down to the primary particle size. This was achieved during the process of seeding the hydrogen fuel using a seeding apparatus that was previously developed ${ }^{2}$. The apparatus is schematically presented in Fig. 2. It basically consisted of a dry bed fluidizer, a fine pickup tube and a particle shearing nozzle. The majority of the fuel passed along circuit $A$ and through the particle shearing nozzle. The flow was accelerated to Mach 3 in the nozzle and at a point upstream of the dual-mode combustor fuel injector, shocked back to subsonic flow. A small amount of fuel bypassed the shearing nozzle and passed through circuit B. This flow was used to levitate the agglomerated particles in the dry bed fluidizer. The levitated agglomerates passed into the pickup tube and were transversely injected into the flow of the particle shearing nozzle. The point of injection corresponded to the point at which Mach 2 flow was attained in the main stream of the shearing nozzle. The interaction of the agglomerates with the fuel stream sheared the agglomerates and dispersed the resulting fine particles into the fuel. This concept is similar to one previously developed for the seeding of air ${ }^{7}$. The flow rate through the fluidized bed was controlled using a remotelyoperated metering valve and this enabled the seeding rate to be controlled during PIV data acquisition. As a means of controlling the fuel total pressure at the seeder outlet, a small amount of fuel bypassed both the shearing nozzle and fluidizer and passed through circuit C. The seeding apparatus was capable of continuous and relatively steady operation for periods approaching 1 hour, after which the fluidized bed could be refilled. At the flow conditions of Table 2, the mass flow rate of hydrogen through the seeder was near $2 \mathrm{gram} / \mathrm{s}$ and the seeding concentration in the dual-mode combustor corresponded to a volume fraction of the order of $10^{-10}$. Since this flow and seeding rate approached the upper limit available from the seeder, the apparatus was not capable of adequately seeding the high mass flux air freestream of the supersonic combustion tunnel.

In order to determine the size of the seeded particles, tests were conducted in which particles were sampled from the dual-mode combustor and then imaged using a scanning electron microscope. The flow conditions of Table 2 and the presence of hydrogen presented difficulties in accurately and safely sampling particles from the test flow. Hence, helium was used to emulate the hydrogen fuel and the combustion tunnel was operated at very low flow velocity. Particles were then manually sampled by placing a small strip of adhesive tape in the flow at the exit of the combustor. The seeder was operated with a flow total pressure near that in Table 2. As the speed of sound was 1.5 times lower for helium than for hydrogen in the particle shearing nozzle, a lower flow velocity was expected. Hence, lower particle shear stresses and higher final particle diameters were anticipated. The scanning electron micrographs of the seeded particles revealed that spherical particles with diameters as low as $0.7 \mu \mathrm{m}$ were produced. Other tests using nitrogen, and conventional (low resolution) imaging of particles while present in the flow in the combustor, confirmed that the output particle diameter monotonically decreased as the gas speed of sound increased. Therefore, it was concluded that the use of hydrogen in the seeder would produce particles with diameters less than $0.7 \mu \mathrm{m}$ and approaching $0.3 \mu \mathrm{m}$.

Given an estimate of seed particle diameter, the ability of the particles to correctly track the flow can be assessed. For measurements in turbulent supersonic flows, two aspects are important. First, the extent of particle lag in response to high frequency turbulence and second, the extent of particle lag following a sudden large scale acceleration or deceleration of the flow. Particle lag is often quantified using the particle relaxation time, $\tau_{p}=\rho_{p} d_{p}^{2} / 18 \mu$, which is based on Stokes' drag of a sphere. Here $d_{p}$ and $\rho_{p}$ is the effective particle diameter and density, respectively, and $\mu$ is the gas viscosity. At lower $\tau_{p}$ a particle is able to accurately respond to turbulence at higher frequency and will more quickly respond to a sudden change in flow velocity ${ }^{19,20}$. Given the complexity of the flow field of the dual-mode combustor, particle lag was assessed for the following representative cases. First, frequency response was estimated for a particle in an air stream and a hydrogen stream at the conditions of Table 2 . The extent of lag downstream of a flow deceleration from these conditions was then estimated. Using the approach of Ref. 19, which is based on the particle relaxation time above, $0.3 \mu \mathrm{m}$ alumina particles are expected to follow a turbulent fluctuation, to within $90 \%$ of the fluid velocity, up to a frequency of at least $30 \mathrm{kHz}$ and $6 \mathrm{kHz}$, for air and hydrogen, respectively.

The extent of particle lag downstream of a flow deceleration was determined using a Stokes' drag coefficient with a compressibility correction ${ }^{20}$. For a step velocity change the particle response is exponential. For example, downstream of an oblique shock, such as one generated by a $10^{\circ}$ ramp in air at the flow conditions of Table 2, a $0.3 \mu \mathrm{m}$ alumina particle will achieve $90 \%$ of the change in flow velocity at a normal distance from the shock of $3 \mathrm{~mm}$. This means that PIV measurements directly downstream of the shock will be biased and the shock will be 'smeared'. However, it is noted that such features can only be resolved to within the spatial resolution of the technique in any case; which for the present experiment is $1.2 \mathrm{~mm}$ 
(as discussed later). For the case of hydrogen flow deceleration, it is noted that when combustion occurs in the dual-mode combustor, heat release lowers local Mach numbers to subsonic levels through a large portion of the duct. In fact, previous CFD analysis ${ }^{2}$ indicated that no shocks were present in the measurement region of interest. However, as shown in Table 2, hydrogen is injected at $1760 \mathrm{~m} / \mathrm{s}$ into an air flow at $1030 \mathrm{~m} / \mathrm{s}$ and, thus, significant flow deceleration is still expected. As noted later and presented in Fig. 9(a), CFD analysis predicted that a gradual mixing of these two velocity fields occurred over a distance of about $60 \mathrm{~mm}$. If instead of a gradual decrease, a conservative approach is adopted and step change in velocity is assumed, then the drag relations of Ref. 20 indicate a $0.3 \mu \mathrm{m}$ alumina particle will recover to within $10 \%$ of $1030 \mathrm{~m} / \mathrm{s}, 6 \mathrm{~mm}$ downstream of the step change. Given the lag length for this worst case scenario is $10 \%$ of the mixing length for a gradual deceleration, it is concluded that particle lag will not be significant for the case of combusting hydrogen-air. For the case of noncombusting hydrogen-air, however, local flow Mach numbers will remain between 1.7 and 2.1 and hence, shocks and expansion waves will be present. Following the previous analysis for an oblique shock, particle lag of the order of $4 \mathrm{~mm}$ can expected in the mixing-only flow.

\section{Light-sheet and imaging}

Light sheet supply and imaging was provided using a commercial system developed by TSI Incorporated. The system consisted of a Spectra Physics dual-cavity Nd:YAG laser, data acquisition personal computer and a TSI digital CCD camera and laser-camera synchronizer. TSI Insight 3.2 software was used for image acquisition and subsequent post-processing. Fig. 3 presents a schematic of the laser sheet, imaging configuration and reference axes. The laser sheet was introduced, through the observation window in the dual-mode combustor, such that it was perpendicular to and on the centerline of the injection wall. The optical axis of the camera was mounted normal to the laser sheet. The output of the two Nd:YAG cavities were combined and frequency doubled to produce a green, $532 \mathrm{~nm}$ collinear beam. Using sheet forming optics, the beam was delivered into the combustor as a $12 \mathrm{~mm}$ wide, $0.8 \mathrm{~mm}$ thick sheet. Each laser cavity produced a pulse with a 10 ns temporal pulse width at an energy near $100 \mathrm{~mJ}$ per pulse. Using a Stanford Research Systems Inc. digital delay/pulse generator, the laser pulses were typically temporally separated by 100 ns. Pairs of light pulses were generated at the laser repetition frequency of 10 $\mathrm{Hz}$ and were synchronized with the camera such that both pulses were captured on a single frame. Double exposure of each frame, rather than the capture of one pulse per frame, was necessary due to limitations in the camera framing rate.

The CCD camera collected light over an active array of $1000 \times 1016$ pixels that were each $9 \times 9 \mu \mathrm{m}$ in size. The camera was operated with a $60 \mathrm{~mm}$ Nikkor lens, at an $f$. number of 16 . The configuration produced a diffractionlimited resolution near $40 \mu \mathrm{m}$ ( 4 pixels) and a field of view near $9.5 \mathrm{~mm}$ square. In order to filter out unwanted luminosity of the combustor and flame, a narrow bandpass filter (centered on $532 \mathrm{~nm}$ ) was mounted on the front of the lens. As the camera field of view was smaller than the total measurement region in the combustor, images were captured at a series of 24 measurement stations. The measurement region extended from $\mathrm{X} / \mathrm{H}=1$ to 13.5 and $\mathrm{Y} / \mathrm{H}=0.7$ to 2.8 . Klinger Scientific translation stages, with a resolution of $1 \mu \mathrm{m}$, were used to traverse the camera and laser sheet over this region. A series of 200 images were captured at each station. Given the imaging rate, flow velocity and the camera field of view, particles imaged in one frame were not present in the next. Hence, velocity measurements determined from each frame were statistically independent in time.

\section{Data processing and validation}

The Insight software was used to determine velocity information using a method of one-frame spatial crosscorrelation on each image. The analysis was optimized according to the recommendations of other investigators ${ }^{21-24}$. As depicted in Fig. 3, each image was divided into an array of $14 \times 7$ interrogation subregions and the mean particle displacement was determined for each region. Based on the time between laser pulses, the mean velocity was calculated. The cross-correlation was performed with a subregion offset that approximately equaled the mean particle displacement of each image. This had the effect of optimizing the detectability of particle displacement and reducing the associated displacement error ${ }^{24}$. Because a double exposed image was used for the cross-correlation, the direction of particle displacement could not be determined. Hence, it was necessary to assume a flow direction. This meant that reverse flow and velocities at or near zero could not be measured ${ }^{25}$. It is noted that CFD predictions for the reacting case (which are presented below) showed no flow reversal in the measurement region of interest. Each subregion was $128 \times 64$ pixels in size and, for the $14 \times 7$ array and image size, this resulted in no subregion overlap. The subregion size corresponded to a $1.2 \times 0.6$ $\mathrm{mm}$ area on the measurement plane in the combustor. For the $100 \mathrm{~ns}$ time delay between laser pulses, particle displacement in the plane of the laser sheet was typically 11 pixels. This is in accord with recommendations of an in-plane displacement of less 
than $1 / 4$ the subregion $\operatorname{size}^{21}$. As the laser sheet was aligned along the axis of symmetry of the combustor, nominal particle displacement out of the plane of the laser sheet was expected to be only dependent on flow turbulence. Assuming a maximum relative turbulence intensity of 1.0 , for example, out of plane RMS displacement is equivalent to 11 pixels or equal to 0.1 $\mathrm{mm}$. Considering the laser sheet thickness is near 0.8 $\mathrm{mm}$, this displacement is less than $1 / 4$ the laser sheet thickness and is in accord with recommendations for maximum out of plane displacement ${ }^{21}$.

In order to maximize detectability of valid particle displacements, a minimum of 10 particle pairs per interrogation subregion is recommended ${ }^{22}$. As only the fuel was seeded in the present experiment, the number of particle pairs per subregion varied with the extent of fuel-air mixing. However, along the center of the fuel plume, throughout the measurement region, 10 particle pairs per subregion were typically observed. At the edge of the fuel plume, particle concentration was lower and this reduced the probability of valid velocity vector generation in this region. In order to further maximize valid displacement detection, a detectability criterion of 1.1 was adopted and this is in accord the recommendation of Ref. 22 .

Due to the stochastic nature of the number of particles per interrogation subregion, some subregions are expected to have a low number of particle pairs and produce spurious vectors ${ }^{26}$. Such vectors are also expected to be generated for subregions where the image has a low signal-to-noise ratio (SNR) ${ }^{27}$. Hence, spurious vectors are most likely to occur along the edge of the fuel plume where the particle density is low, or outside the plume where the density is zero. In order to remove spurious and outlying velocity vectors, each single generated array of vectors was first passed through a global range filter. Any vector with an axial component of more than $3000 \mathrm{~m} / \mathrm{s}$ or a transverse component of more than $\pm 1500 \mathrm{~m} / \mathrm{s}$ was removed. Remaining outliers that were more than two standard deviations from the mean velocity of each array were then removed using a global standard deviation filter. Further vector removal was necessary because the CCD camera introduced noise on the last four pixel columns in each image. This lowered the SNR of affected subregions to the extent that significant numbers of spurious vectors were generated. In order to account for this noise, the last column of vectors was removed from the generated $14 \times 7$ array.

Vectors removed during the validation process were not replaced using interpolation or other data filling techniques. For a given point in the combustor, the validation procedure removed between $1 \%$ and $100 \%$ of vectors. The removal rate was $100 \%$ outside the fuel plume where the particle density was zero and vector generation was based on background noise (and along the last column of the subregions arrays where the SNR was high). It is noted that the range and standard deviation filters are expected to remove a high proportion of spurious or outlying vectors, but not all.

Following vector validation, the time-averaged velocity for each subregion was determined by averaging over the sample of 200 images that were acquired at each measurement station. The magnitude of time-averaged streamwise velocity, $\bar{V}$, was determined for each subregion from $\bar{V}=\sqrt{\bar{u}^{2}+\bar{v}^{2}}$, where $\bar{u}$ and $\bar{v}$ are the time-averaged $X$ and $Y$ components of velocity, respectively, for each subregion.

\section{Error analysis}

Velocity measurement uncertainty, spatial accuracy and biasing due to non-uniform seeding is now addressed. As the mean velocity of each interrogation subregion was determined using the mean particle displacement and time between laser pulses, the uncertainty in velocity depends on the uncertainty in displacement and in pulse delay. The uncertainty in displacement is a function of the uncertainty due to the cross-correlation method and in image magnification. The accuracy of cross-correlation methods have been extensively studied by others ${ }^{11,21-25}$. It has been shown that interpolation, inherent in the analysis, results in particle displacement errors of less than one pixel ${ }^{23}$. It has also been demonstrated that if the particle displacement is larger than $1 / 2$ pixel, then the error is practically independent of the displacement $t^{24}$. Ref. 24 has determined the displacement error for the case of cross-correlation of two single exposure images. For the imaged pair density and particle image and pixel size of the present experiment, a maximum error near 0.1 pixel would be expected for such a cross-correlation. Ref. 25 has shown that for a given particle image diameter, subregion size and image density, cross-correlation of double exposure, single frame images (as used in the present experiment), results in displacement errors that are about six times that for two single exposure images. Hence, a conservative displacement error of six times the above error, or 0.6 pixels, was adopted for the present experiment. This displacement error is further increased if there is a velocity gradient across the interrogation subregion. The velocity gradients predicted by CFD for the dual-mode combustor with combustion of hydrogen for example, would result in a displacement error of 0.35 pixels $^{22}$. When this is combined with the above error of 0.6 pixels as a root sum square (assuming these errors are normally distributed and independent), a 
displacement error near 0.7 pixels is established. This error represents $6 \%$ of the typical measured displacement of 11 pixels. For the case where combustion is precluded in the dual-mode combustor, velocity gradients of shocks and expansion waves are expected to drive this error higher in some interrogation subregions.

The uncertainty in time between laser pulses was estimated to be $1 \%$ (attributable to laser pulse jitter) and the uncertainty in magnification was regarded as negligible. Therefore, combining the pulse uncertainty with the displacement error of $6 \%$, again as a root sum square (RSS), results in a total uncertainty in measured velocity, $\sigma_{v}$, near $6 \%$. Particle lag is expected to further increase this error immediately downstream of regions of sudden flow acceleration or deceleration. However, as previously discussed, this is expected to be small for the combustion case.

In determining the average velocity magnitude, $\vec{V}$, the statistical uncertainty in the mean, $\sigma_{\bar{v}}$,was determined from $\sigma_{\bar{v}}=1.96 \hat{\mathrm{s}} / \sqrt{\mathrm{n}}$ (uncertainty for a $95 \%$ confidence interval with negligible systematic uncertainties) ${ }^{2 a}$. Here $\hat{s}$ is the relative standard deviation, which is given by $\hat{s}=s / \bar{V}$, where $s$ is the sample standard deviation; $s=\sqrt{s_{u}^{2}+s_{v}^{2}}$. The variables $s_{v}$ and $s_{v}$ are the corresponding standard deviations for the $u$ and $v$ components of velocity and $n$ is the number of vectors that passed the validation procedure. Assuming particle lag and other error sources are low, Ref. 28 explains that that the relative standard deviation can be related to the velocity measurement error and flow relative turbulence intensity, $\sigma_{\mathrm{T}}$, by $\hat{\mathrm{s}}^{2}=\sigma_{\mathrm{v}}^{2}+\sigma_{\mathrm{T}}^{2}$. Here it is assumed that the contribution of any spurious vectors to the sample standard deviation is low (i.e. spurious vectors that possibly pass the vector validation filters). From knowledge of $\hat{\mathbf{s}}$ and $\sigma_{v}$, the turbulence intensity can thus be determined.

The uncertainty of the position of each subregion in space was determined by considering the errors inherent in operating the camera and laser sheet optics on translation stages. Each translation stage had a resolution of $1 \mu \mathrm{m}$ and was zeroed to a nominal reference point to within $0.2 \mathrm{~mm}$. The stages were estimated to track along the axes of the dual-mode combustion to within $0.5 \mathrm{~mm}$. Combining these errors as a RSS, and considering further that the error in image magnification was small, a spatial error near $0.5 \mathrm{~mm}$ was adopted.
Because the fuel of the dual-mode combustor was seeded and the free-stream was not, velocity biasing due to non-uniform seeding is possible. Ref. 10 explains that this biasing occurs in the mixing field of a two-stream flow when one stream is seeded and the velocity of each stream is significantly different. Because the velocity is determined from imaged particles, an average velocity determined from a series of images of the mixing region may be biased towards the velocity of the stream that is seeded. This occurs because only fluid structures that emanate from the seeded flow contain particles that are included in the calculation of the average velocity. If a structure from the unseeded stream is present in an image at any one instant in time, it is not included in the determined mean velocity. The effect is common to all particle-based velocimetry techniques ${ }^{10}$. Seeding biasing has been observed in PIV measurements of planar turbulent mixing wakes ${ }^{16}$. In that experiment, a subsonic jet was seeded and time-averaged velocity measurements were obtained with and without seeding of a supersonic co-flowing stream. The velocity in the middle of the wake was the same for both cases; however, biasing was noted along the edges of the mixing wake. The extent of biasing would appear to be case specific, however. PDV measurements of a Mach 0.85 jet issuing into a low speed stream exhibited no velocity biasing. In that experiment, the jet was seeded and time-averaged velocity profiles at 6 nozzle diameters downstream of the nozzle were the same with and without co-flow seeding. Therefore, it can be concluded that for the present experiment, if biasing does indeed occur, it may be expected to be limited to along the outer edges of the mixing and combustion wake.

\section{Results and Discussion}

\section{Pressure distribution}

Measured wall pressure distributions are presented in Fig. 4 for the case of fuel-off, mixing fuel-on and reacting fuel-on. The measurements are displayed as a function of distance from the ramp base and have been normalized by the measured fuel-off facility nozzle exit static pressure. The location of major geometry changes of the combustor are marked in the figure. For the fueloff case, a pressure rise on the face of the ramp, behind the ramp-induced shock, is evident near $-5 \mathrm{H}$. The pressure then falls in the ramp base flow region. Downstream of the base, ramp-generated shock and expansion waves lead to significant variations in the streamwise pressure distribution. At $40 \mathrm{H}$, the pressure then rises to the atmospheric exit condition. The pressure levels throughout the combustor are consistent with the bulk of the flow remaining supersonic. For the mixing case, when fuel is injected but not manually 
ignited, the pressure distribution remains largely unchanged. The pressure levels are again consistent with the bulk of the flow remaining supersonic. As the injected fuel stream is also supersonic, flow in the PIV measurement region, between $1 \mathrm{H}$ and $13.5 \mathrm{H}$, is thus expected to be supersonic.

Referring to the case of reacting fuel-air, it is evident that combustion produces a significant rise in wall pressures. The pressure on the ramp face and at the nozzle exit, however, remain unaltered. This indicates that there is little or no influence of combustion upstream of the ramp base and that the inflow to the isolator remains supersonic. Downstream of the ramp, the pressure rises to over two times the nozzle exit pressure. The absence of significant variations in streamwise pressure in this region indicates that a zone of low Mach number flow exists near the wall. This is consistent with previous experimental and numerical observations $^{2}$. The Mach number in the PIV measurement region is, therefore, expected to be low for the reacting fuel-air case.

\section{Velocity measurements}

Fig. 5 presents the measured centerplane distribution of time-averaged velocity. The results are displayed in the form of vector fields, with components of $\bar{u}$ and $\bar{v}$, and contours of streamwise velocity, $\bar{V}$. The results are presented separately for the mixing and reacting cases. Each distribution is a juxtaposition of the time-averaged velocity field determined for each measurement station. No averaging or interpolation has been applied where the image from adjacent measurement stations overlap. As a consequence, the vector concentration can be seen to be higher in the overlap regions. It can be seen that the PIV data processing and validation technique produces good vectors through the middle of the fuel plume. However, the low concentration of vectors and presence of spurious vectors at the edges of the plume reflects the low seed density where the seeded fuel and unseeded freestream mix.

The measurements for the reacting case are also compared with a CFD result in Fig. 5. The simulation was performed using the GASP code and is fully described eleswhere ${ }^{2}$. Ref. 2 found that the numerical model was capable of only approximating the combustor axial pressure distribution and fuel-air mixing and combusting plume when combustion occurred. In the present study, the ability of the model to match the centerplane velocity distribution is assessed. The numerical results of Fig. 5 were obtained for flow conditions similar to those of Table 2, except the air total pressure was $15 \%$ lower for the CFD analysis. The effect of this difference is expected to be minimal. It is noted, however, that the modeling was performed with a fuel injector port having a square cross section (in order to simplify grid generation). This may be expected to alter the shape of the fuel jet immediately downstream of the fuel injector, but have less of an effect on the far field mixing and combustion wake. The numerical results are compared with experiment below.

Fig. 6 presents a contour map of the number of vectors, $n$, used to obtain the time-averaged velocity results of Fig. 5. The maximum vector count equals the number of images acquired at each measurement station, which is 200 . It can be seen that there are generally more vectors along the middle of the plume. Higher numbers of vectors are generated in regions where vector detectability is high and vector validation rejection is low. This occurs where the seed density and signal-tonoise ratio is high. The location of the high count core is consistent with the location of the peak intensity in crossflow fuel plume images that were reported in Ref. 2. It can also be seen in Fig. 6 that the vector count is lowest along the edges of the fuel plume, where particle concentration is expected to be low. This is consistent with the location of spurious vectors and low vector concentration in Fig. 5.

To elucidate the differences in the velocity measurements, the streamwise velocity contours of Fig. 5 are presented in Fig. 7 but with the vectors replaced with interpolated streamlines. Examining the measured velocity contours for the mixing case, it is evident that there is a high speed core in the jet and this extends to $\mathrm{X} / \mathrm{H} \approx 4$. At this point the velocity is close to $75 \%$ of the peak velocity measured on the $\mathrm{X} / \mathrm{H}=1$ plane of 1840 $\mathrm{m} / \mathrm{s}$. It is interesting to note that this peak velocity is not far from the calculated fuel injection velocity of 1760 $\mathrm{m} / \mathrm{s}$. It is noted, however, that the fuel is injected as an underexpanded jet, and hence, the flow velocity at $\mathrm{X} / \mathrm{H}=1$ is not necessarily expected to match the initial injection velocity. Downstream of $X / H \approx 4$, the mixing fuel-air wake approaches the calculated freestream air velocity of $1030 \mathrm{~m} / \mathrm{s}$.

For the reacting case, the peak measured velocity at $\mathrm{X} / \mathrm{H}=1$ was $1750 \mathrm{~m} / \mathrm{s}$ and this is relatively close to that measured for the mixing case. However, the measurements exhibit a high speed core in the fuel jet that extends further downstream from the ramp injector than for the mixing case. The jet reaches $75 \%$ of the peak $\mathrm{X} / \mathrm{H}=1$ velocity near $\mathrm{X} / \mathrm{H}=6$. A similar effect has been observed in subsonic hydrogen jet flames by Ref. 29. Using Laser Doppler Velocimetry, the investigators observed a slower streamwise decay in centerline velocity for a reacting (nitrogen diluted) hydrogen jet in an air co-flow when compared to a non-reacting jet. The 
investigators postulated that the difference was due to temperature rise induced expansion in the flame. This density reduction maintained streamwise velocities and reduced entrainment of the lower speed co-flow into the fuel jet. Ref. 29 also measured reduced turbulence intensities near the jet nozzle, and hence, partly attributed reduced entrainment to reduced turbulent mixing. Although fuel injection in the present experiment is supersonic and that of Ref. 29 was subsonic, the effect of heat release on velocity decay is strikingly similar. It is also noted, in the present case, fuel-air mixing is enhanced via two counter rotating vortices that are generated by the ramp fuel injector. Therefore, it is also possible that combustion reduces the extent of vortex induced turbulent mixing of the lower speed air into the fuel plume.

Downstream of $\mathrm{X} / \mathrm{H}=6$ and at high $\mathrm{Y} / \mathrm{H}$, the velocities for the reacting case are $\sim 20 \%$ lower than for the mixing case. However, velocities are even lower close to the injection wall. The velocity reduction for the reacting case is attributed to the heat release of combustion leading to thermal choking. As previously discussed, Gauba et al. measured time-averaged velocity in the same combustor using the Doppler shift of OH PLIF (at test conditions that approximated those of Table 2). The investigators also observed reduced velocities at high $\mathrm{X} / \mathrm{H}$. Because the technique relied on the imaging of combustion-generated $\mathrm{OH}$, however, the measured velocity field only spatially overlapped with the present data along $\mathrm{Y} / \mathrm{H}=1$ between $\mathrm{X} / \mathrm{H}=8$ and 10 . Along this line, Gauba et al. 4 measured velocities in the range of 200 to $400 \mathrm{~m} / \mathrm{s}$. This compares favorably with the present experimental results in which time-averaged velocities in the range of 200 to $490 \mathrm{~m} / \mathrm{s}$ were measured.

Comparing the measurements for the reacting case with the numerical results, velocity magnitudes can be seen to be generally similar. In the freestream above the fuel jet, the numerical model predicts that thermal choking reduces the flow velocity to between 700 and $850 \mathrm{~m} / \mathrm{s}$. This would appear to be in agreement with the (nonspurious) measurements at low $\mathrm{X} / \mathrm{H}$ along the top of the fuel jet. The axial extent of the high speed core is also similar, however, the penetration angle is different. The numerically predicted peak velocity at X/H=1 of 2095 $\mathrm{m} / \mathrm{s}$ is also higher than the measured level. This difference partially reflects a fuel injection velocity for the numerical model that was $7 \%$ higher than calculated for experiment $(1760 \mathrm{~m} / \mathrm{s})$. Further differences may arise because of differences between experimental and CFD combustor pressures in the vicinity of the expanding underexpanded jet. It is noted however, that as the numerical simulation was performed with a square fuel injector port, a detailed comparison close to the fuel injector is precluded.
Referring to the interpolated streamlines of Fig. 7 for the mixing case, the flow initially lifts away from the injection wall at a penetration angle near $11^{\circ}$. At $\mathrm{X} / \mathrm{H}=3$ to 4 the flow turns back towards the injection wall so that it is almost parallel to the wall. The flow then turns away from the injection wall at $\mathrm{X} / \mathrm{H}=7$ to 8 . For the reacting case, the flow penetrates at a similar angle as for the mixing case. However, the reacting jet maintains this angle further from the ramp base and hence penetrates further into the flow. The streamlines continue to lift off the injection wall until $\mathrm{X} / \mathrm{H}=6$ where the streamlines closest to the injection wall turn back towards the wall. The streamlines at high $\mathrm{Y} / \mathrm{H}$, however, continue to lift off the wall. For both the mixing and reacting cases, the initial penetration angle is close to the fuel injection angle of $10^{\circ}$. The CFD predicted streamlines for the reacting case show the penetration angle to be closer to $7^{\circ}$, which is lower than that measured (and lower than the injection angle of $10^{\circ}$ ). The numerically predicted streamlines also tend to converge rather than diverge. These observations are consistent with previous findings in which the numerically predicted fuel plume penetration and size was smaller than that measured for the reacting case ${ }^{2}$. Although the penetration angle and divergence is lower, the stream lines do turn back towards the injection wall in a manner that is similar to streamlines at mid $\mathrm{Y} / \mathrm{H}$ in the experiment.

Figure 8 presents contour plots of measured relative turbulence intensity (RMS velocity fluctuations normalized by $\bar{V}$ ) for the mixing and reacting cases. The turbulence intensity is calculated from the measured relative standard deviation of velocity using $\hat{\mathbf{s}}^{2}=\sigma_{\mathrm{v}}^{2}+\sigma_{\mathrm{T}}^{2}$ with $\sigma_{\mathrm{v}}=0.06$. Results are only displayed where the vector count is 2 or more. For the mixing case, the turbulence intensity is relatively uniform through the flow. The fuel jet core has approximately the same turbulence intensity as the downstream mixing wake. This level is generally near $10 \%$. Comparing the reacting results with the mixing results, the reacting turbulence intensity is similar to mixing levels through the high speed core of the jet. This low turbulence core extends to $\mathrm{X} / \mathrm{H}=4$ where it terminates at $\mathrm{X} / \mathrm{H}=6$ in a tapering fashion. Downstream of the low turbulence region, the heat release of combustion results in significantly higher turbulence levels, reaching five to six times the mixing levels in some regions. Ref. 29 also reported that combustion significantly increased turbulence levels in the far field of the jet wake. However, as previously mentioned, Ref. 29 found that combustion reduced turbulence levels close to the jet nozzle. This is in contrast to the present measurements. The difference suggests that the slower 
streamwise decay in fuel jet velocity observed in the present study is not due to reduced turbulent mixing.

In order to facilitate a direct comparison between the mixing and reacting results, and account for the difference in penetration angle between experiment and $C F D$, velocity and turbulence intensity levels were extracted along given streamlines. A single streamline that represented the center of the high speed core was chosen for each case. Each streamline lay between the streamlines marked as A and B in Fig. 7. In order to account for the overlap between adjacent measurement stations, the data were first averaged and linearly interpolated onto a rectangular array. Quantities were then extracted along the interpolated streamline at regular intervals. Fig. 9(a) presents the measured timeaveraged velocity along each streamline for the mixing and reacting cases, as a function of the distance downstream of the fuel injector, X. As can be observed in Fig. 7 , the velocity is initially the same for both cases. The reacting flow then slightly accelerates. The heat release of combustion maintains higher centerline velocities through to $-6 \mathrm{H}$. Downstream of this point, thermal choking dominates the reacting flow velocity and the level drops below that for mixing only. Also presented in Fig. 9(a) is the numerically predicted velocity along a reacting core streamline. The CFD results are generally $20 \%$ higher than for experiment.

Measured turbulence intensities, on the interpolated streamlines, for the mixing and reacting cases are presented in Fig. 9(b). Similarly to Fig. 8, the turbulence intensities for the two cases are initially close up to $4 \mathrm{H}$. At this point there is a significant increase in turbulence intensity for the reacting case, reaching 4 times the mixing levels in this case. Interestingly, the reacting and mixing turbulence intensities start to converge at high $\mathrm{X} / \mathrm{H}$.

\section{Conclusions}

Velocity measurements in a model dual-mode scramjet combustor have been obtained using Particle Image Velocimetry (PIV). The experiment consisted of the direct connect operation of a Mach 2 hydrogen-air combustor with a single unswept ramp fuel injector. Alumina particles were seeded into the fuel stream and PIV was performed on the mixing and combustion wake of the fuel injector. In order to investigate the effects of combustion on the fuel-air mixing, measurements were conducted for mixing (non-reacting) and reacting hydrogen-air.

When seeding a helium stream, the seeding apparatus was shown to be capable of producing seed particles with diameters down to $0.7 \mu \mathrm{m}$. Diameters as small as
$0.3 \mu \mathrm{m}$ were expected when the apparatus was applied to the hydrogen fuel stream. The latter particles were expected to be capable of tracking flow turbulence at frequencies to at least $6 \mathrm{kHz}$. Some particle lag was expected immediately downstream of shocks for the mixing case. However, for the reacting case, thermal choking was expected to significantly reduce streamwise velocity gradients in the flow and particle lag was predicted to be minimal. The flow velocity was determined by performing digital cross-correlation analysis of double exposed images of illuminated seed particles. The measurements had a spatial resolution in the $x, y$ and out of plane directions of $1.2,0.6$ and 0.8 $\mathrm{mm}$, respectively. Experimental uncertainty in instantaneous velocity was estimated to be near $6 \%$.

On the centerplane of the combustor, significant differences in the time-averaged velocity and turbulence intensity distributions were observed for the mixing and reacting cases. In the near field of the fuel jet, a slower streamwise decay of velocity was observed along the axis of the jet for the reacting case than for the mixing case. This slower velocity decay is even more significant when it is considered that the difference between the fuel injection velocity and the freestream velocity is greater for the reacting case than for the mixing case (based on CFD, thermal choking is expected to reduce the air freestream velocity to $70-80 \%$ of the mixing levels). In the far field of the fuel plume, downstream of $X / H=4-6$, a velocity reduction due to thermal choking was observed. In terms of penetration into the freestream and away from the injection wall, penetration angles were observed to be close to the jet injection angle for both the mixing and reacting cases. However, the reacting jet penetrated further into the freestream. The measured penetration angles were higher than that predicted by CFD for the reacting case.

Turbulence levels in the non-reacting jet and in the mixing wake were found to be relatively uniform. However, the heat release of combustion was found to significantly increase turbulence levels for the reacting case in the far field of the plume. In the near field, turbulence levels were similar to the mixing levels. Along a given streamline, the change in turbulence level was abrupt between these two fields. This sudden increase in turbulence suggests that increased turbulent entrainment of air into the fuel plume may also abruptly occur.

The present study has demonstrated the successful application of PIV to the supersonic combustion field of a dual-mode scramjet. While a lack of co-flow seeding meant that velocity could only be measured in the fuel plume and particle size and extent of particle lag were not fully defined, it was established that the technique 
was capable of generating accurate measurements in such a flow. The differences observed in the fuel plume between the mixing and reacting cases illustrate the need to validate dual-mode combustor computational codes against reacting flow field data and not just mixing data. In terms of the measured magnitude of time-averaged velocity, the level of agreement with a sample computational solution was encouraging for the reacting case. It is anticipated that measurements of turbulence intensity, for example, can be used to improve turbulence models and hence further improve CFD predictive capabilities.

\section{Acknowledgements}

This work was sponsored by NASA through grants NAG-1-2085 and NAG-1-2131. C.R. McClinton and D.E. Reubush of the NASA Langley Research Center contributed as technical monitors. The authors also appreciate the contribution of C.G. Rodriguez of the National Research Council, Hampton, VA, for performing the numerical simulation, and the assistance of Tim Quagliaroli during installation and commissioning of the PIV system.

\section{References}

'McClinton, C.R., Holland, S.D., Rock, K.E., Engelund, W.C., Voland, R.T., Huebner, L.D., and Rogers, R.C. "Hyper-X Wind Tunnel Program," AIAA Paper 98-0553, Jan.1998.

${ }^{2}$ Goyne, C.P., McDaniel, J.C., Quagliaroli, T.M., Krauss, R.H., and Day, S.W., "Dual-Mode Combustion of Hydrogen in a Mach 5, Continuous-Flow Facility," accepted for publication in Journal of Propulsion and Power, 2001.

${ }^{3}$ Krauss, R.H., Gauba, G., Whitehurst, R.B., Quagliaroli, T.M., and Laufer, G., "Experimental and Numerical Investigation of Steam-Vitiated Supersonic Hydrogen Combustion," AIAA Paper 96-0856, Jan. 1996.

${ }^{4}$ Gauba, G., Klavuhn, K.G., McDaniel, J.C., Victor, K.G., Krauss, R.H., and Whitehurst, R.B., "OH Planar Laser-Induced Flourescence Velocity Measurements in a Supersonic Combustor," AlAA Journal, Vol. 35, No. 4, 1997, pp. 678-686.

${ }^{5}$ Rodriguez, C.G., White, J.A., and Riggins, D.W., "Three-Dimensional Effects in Modeling of Dual-Mode Scramjets," AIAA Paper 2000-3704, July, 2000.

"Miles, R.B., and Lempert, W.R., "Quantitative Flow Visualization in Unseeded Flows," Annu. Rev. Fluid :Wech., 29, pp.285-326, 1997.

${ }^{7}$ Smith, M.W., "Application of a Planar Doppler Velocimetry System to a High Reynolds Number Compressible Jet," AIAA Paper 98-0428, Jan. 1998.
'Meyers, J.F., and Komine, H., "Doppler global velocimetry: a new way to look at velocity," Laser Anemometry, Vol. 1, 1991, pp. 289-296.

${ }^{9}$ Adrian, R.J., "Particle-imaging techniques for experimental fluid mechanics," Annu. Rev. Fluid Mech, 23, 1991, pp. 261-304.

${ }^{10}$ Samimy, M., and Wernet, M.P., "Review of Planar Multiple-Component Velocimetry in High-Speed Flows," AlAA Journal, Vol. 38, No. 4, 2000, pp. 553574.

"Willert, C.E., and Gharib, M., "Digital particle image velocimetry," Experiments in Fluids, 10, 1991, pp. 181-193.

${ }^{12}$ Lawson, N.J., Page, G.J., Halliwell, N.A., and Coupland, J.M., "Application of Particle Image Velocimetry to a Small-Scale de Laval Nozzle," AIAA Journal, Vol. 37, No. 7, 1999, pp. 798-804.

${ }^{13}$ Haertig, J., Havermann, M., Rey, C. and George, F., "PIV Measurements in Mach 3.5 and 4.5 Shock Tunnel Flow," AIAA Paper 2001-0699, Jan. 2001.

${ }^{14}$ Humphreys, W.M., Bartram, S.M., and Blackshire, J.L., "A survey of particle image velocimetry applications in Langley aerospace facilities," ALAA Paper 93-0411, Jan. 1993.

${ }^{15}$ Mugal, M.G., Lourenco, L.M., and Krothapalli, A., "Instantaneous Velocity Measurements in Laminar and Turbulent Premixed Flames Using On-Line PIV," Combust. Sci. and Tech., Vol 106, 1995, pp.239-265.

${ }^{16}$ Nakagawa, M., and Dahm, W.J.A., "Velocity Measurements in the Near-Field of Supersonic Planar Turbulent Wakes," AIAA Paper 2000-2229, June 2000.

${ }^{17}$ Krauss, R.H., McDaniel, J.C., Scott J.E., Whitehurst, R.B., Segal, C., Mahoney, G.T., and Childers, J.M., "Unique, clean-air, continuous-flow, high-stagnation-temperature facility for supersonic combustion research," AIAA Paper 88-3059, July, 1988.

${ }^{18}$ Krauss, R.H., and McDaniel, J.C., "A Clean Air Continuous Flow Propulsion Facility," AIAA Paper 923912 , July, 1992.

${ }^{19}$ Melling, A., "Tracer particles and seeding for particle image velocimetry," Meas. Sci. Technol., 8, 1997, pp. 1406-1416.

${ }^{20}$ Meyers, J.F., "Generation of Particles and Seeding, Laser Velocimetry," Lecture Series 1991-08, von Karman Institute for Fluid Dynamics, Belgium, June 1991.

${ }^{21}$ Keane, R.D., and Adrian, R.J., "Optimization of particle image velocimeters. Part I: Double pulsed systems," Meas. Sci. Technol., 1, 1990, pp. 1202-1215.

${ }^{22}$ Keane, R.D., and Adrian, R.J., "Theory of crosscorrelation analysis of PIV images," Applied Scientific Research, 49, 1992, pp. 191-215.

${ }^{23}$ Westerweel, J., "Fundamentals of digital particle image velocimetry," Meas. Sci. Technol., 8, 1997, pp. 1379-1392. 
${ }^{24}$ Westerweel, J., Dabiri, D., and Gharib, M., "The effect of a discrete window offset on the accuracy of cross-correlation analysis of digital PIV recordings," Experiments in Fluids, 23, 1997, pp. 20-28.

${ }^{25}$ Raffel, M., Willert, C.E., and Kompenhans, J., "Particle Image Velocimetry, A Practical Guide," Experimental Fluid Mechanics, edited by R.J. Adrian, M. Gharib, W. Merzkirch, D. Rockwell and J.H. Whitelaw, Springer-Verlag, Berlin, 1998.

${ }^{26}$ Westerweel, J., Draad, A.A., van der Hoeven, J.G.Th., and van Oord, J., "Measurement of fullydeveloped turbulent pipe flow with digital particle image velocimetry," Experiments in Fluids, 20, 1996, pp. 165-177.

${ }^{27}$ Nogueira, J., Lecuona, A., and Rodriguez, P.A., "Data validation, false vectors correction and derived magnitudes calculation on PIV data," Meas. Sci. Technol., 8, 1997, pp.1493-1501.

${ }^{23}$ Wernet, M.P., "Development of digital particle imaging velocimetry for use in turbomachinery," Experiments in Fluids, 28, 2000, pp. 97-115.

${ }^{29}$ Takagi, T., Shin, H., and Ishio, A., "Properties of Turbulence in Turbulent Diffusion Flames," Combustion and Flame, 40, 1981, pp. 121-140. 

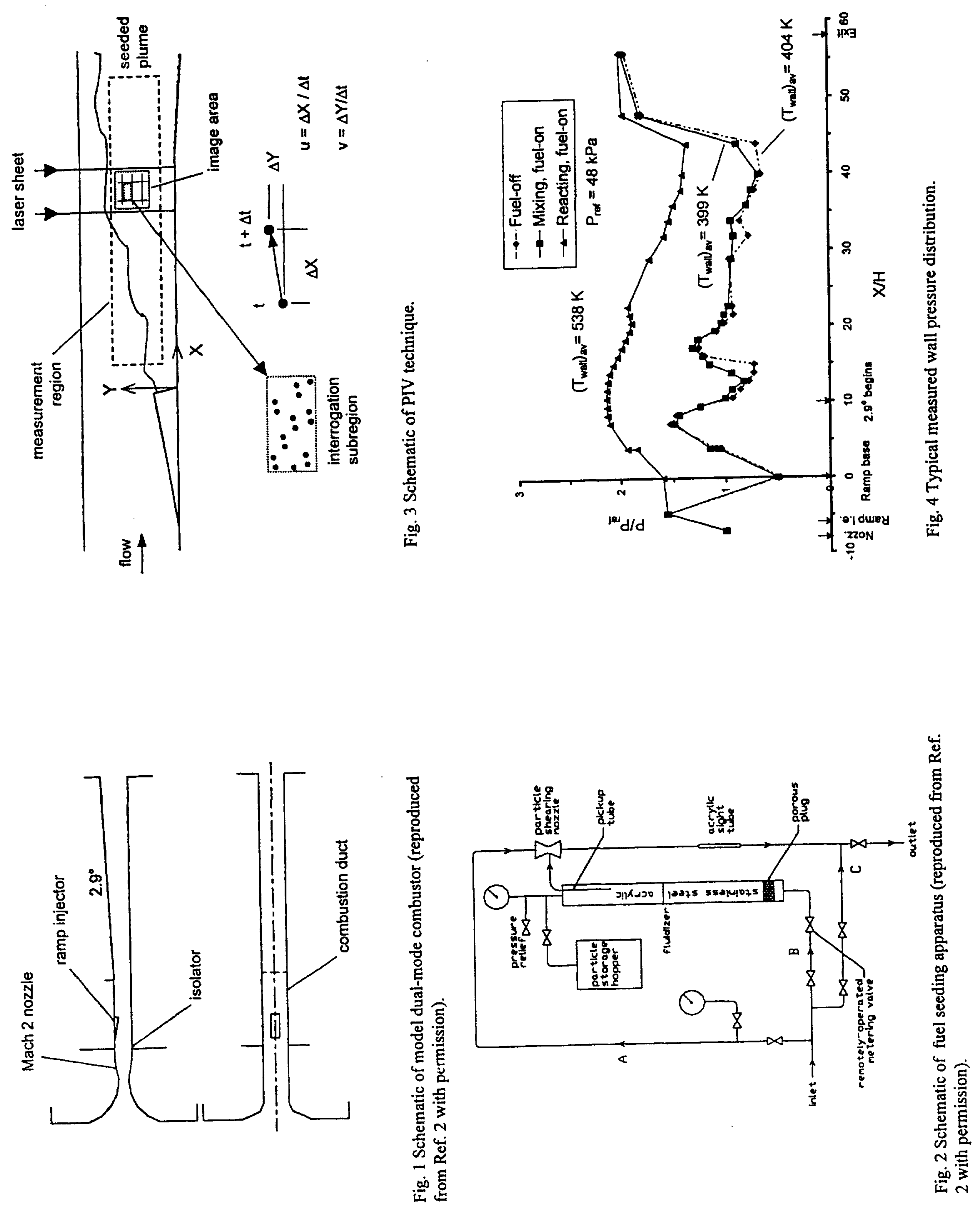

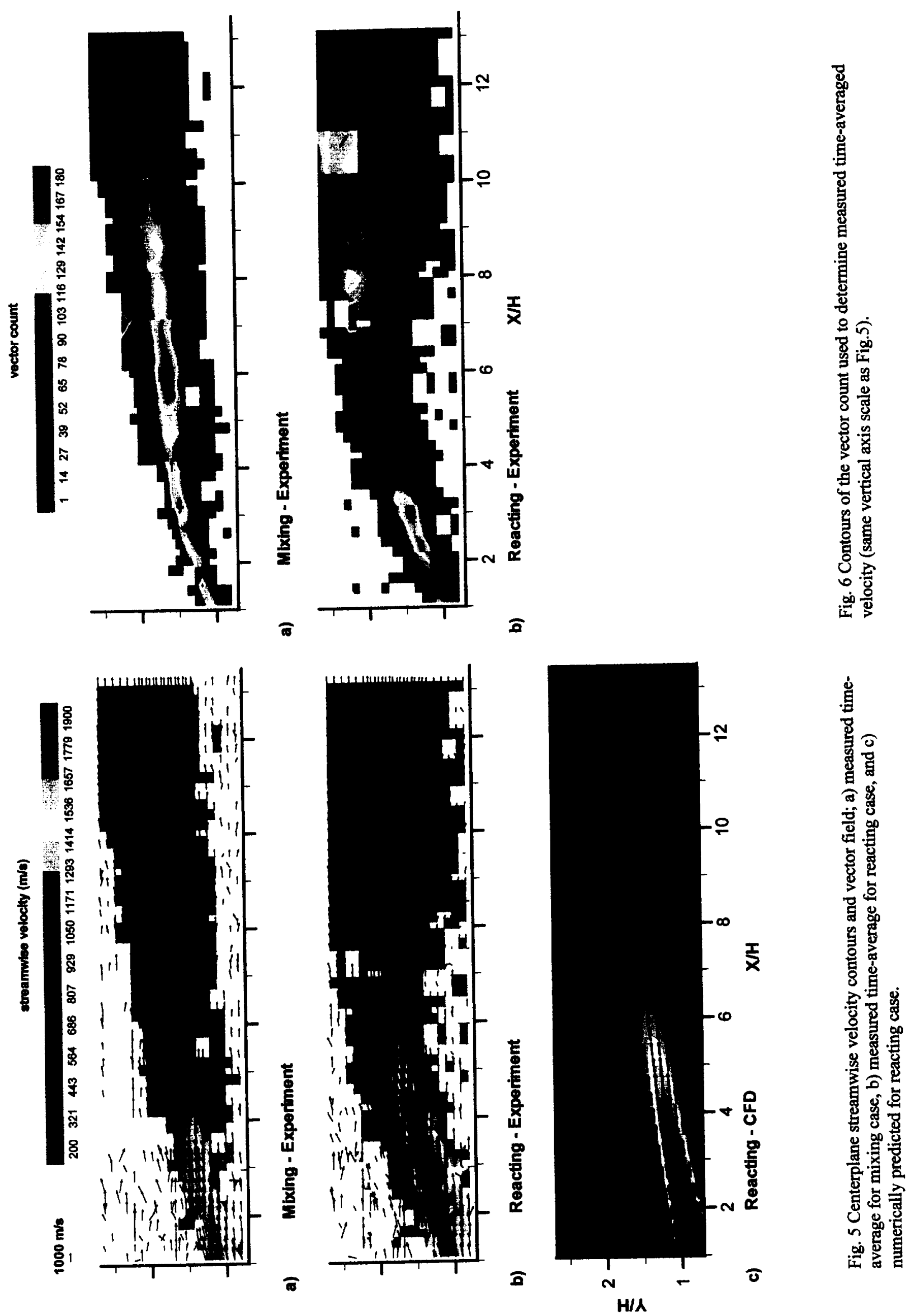

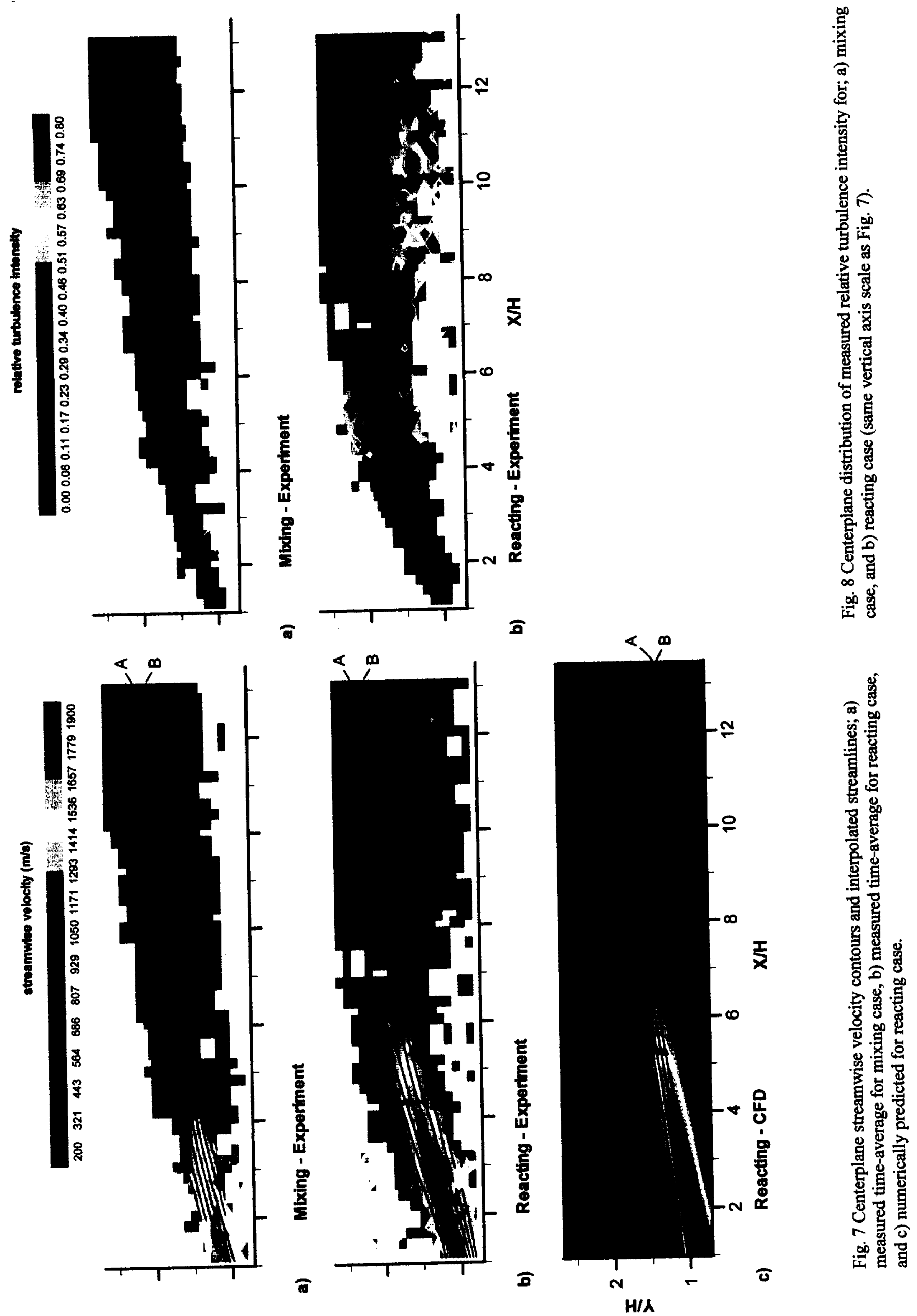

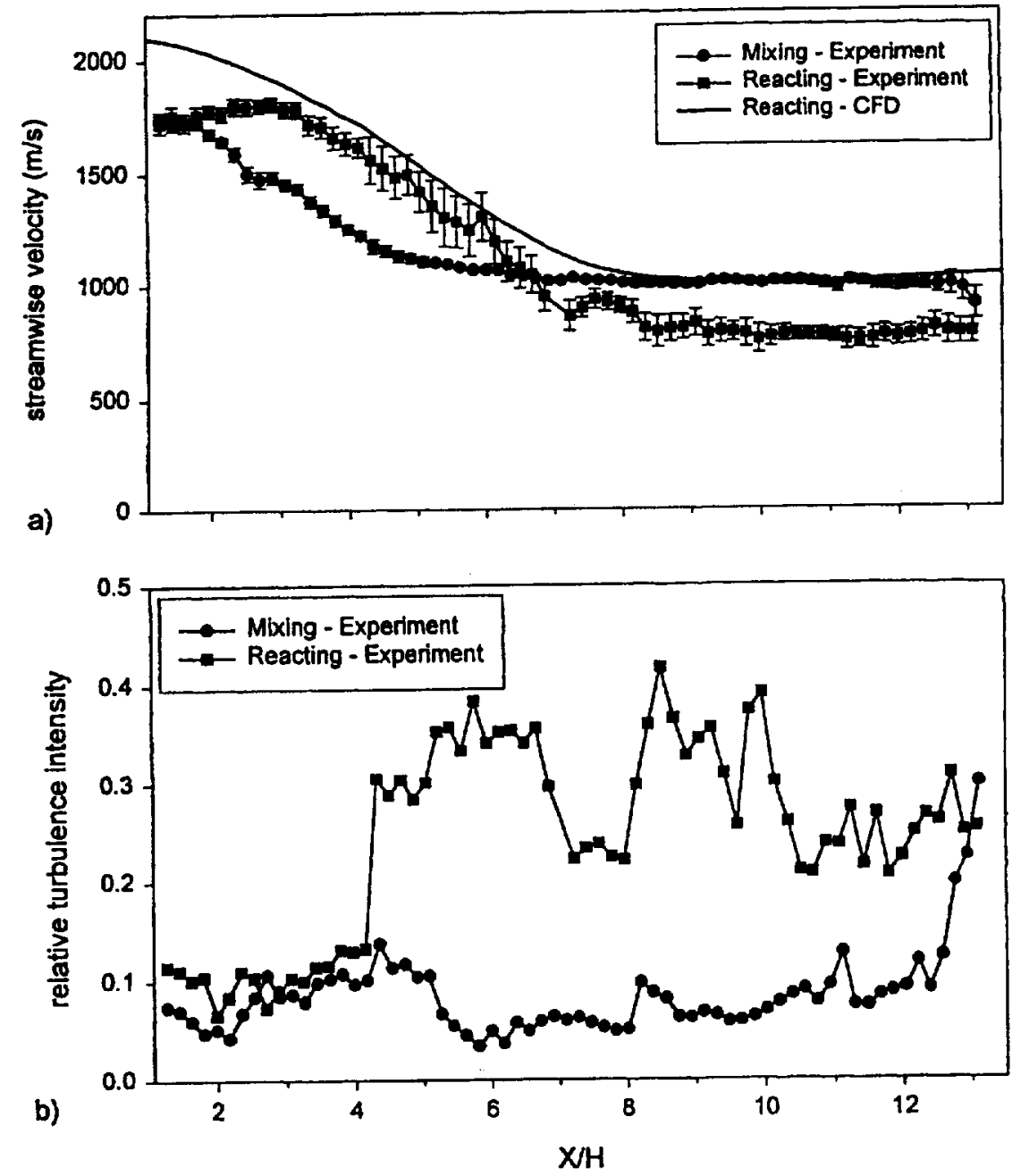

Fig. 9 Plume axial distribution of ; a), measured time-average of streamwise velocity, and comparison with CFD, b) measured relative turbulence intensity. 